\title{
Réflexions sur l'ordre des mots en français (les constituants majeurs de l'énoncé)
}

\author{
Claude Muller \\ Claude.Muller@u-bordeaux3.fr
}

\section{Introduction}

On fera ici le point sur une des questions majeures de la syntaxe du français: la disposition des constituants les uns par rapport aux autres. On s'intéressera seulement aux constituants majeurs de l'énoncé, en laissant de côté l'analyse interne des syntagmes, notamment des syntagmes nominaux.

L'étude de l'ordre des mots est un des domaines majeurs de la syntaxe. Longtemps dominé par des considérations idéologiques sur le rapport avec un "ordre de la pensée" décrit comme universel, l'ordre des mots du français a fait l'objet d'une investigation systématique à l'époque moderne. Un travail pionnier qui reste une source d'inspiration pour la richesse de ses données est le livre de Blinkenberg (1928). Parmi les grammaires, il faut surtout prendre en compte Damourette \& Pichon (1911-1940), notamment les chapitres du volume IV sur la "rétrogression" (les inversions du sujet). Pour les théories contemporaines, Kayne 1973 est un texte fondateur, qui fixe une terminologie toujours utilisée à l'heure actuelle. D'autres approches ont joué un rôle majeur dans ce domaine: l'approche topologique est à l'origine du travail en diachronie de Skårup (1975) qui reste une référence majeure pour l'ancien français. Pour le français moderne, dans une approche contrastive, Herslund (2006), et pour une présentation des analyses typologiques, Gerdes \& Muller (2006). Plus récemment, d'autres théories se sont intéressé à l'ordre des mots en français (voir entre autres Bonami \& Godard 2001 pour HPSG, sur l'inversion du sujet nominal; Kampers-Mahne et alii 2004, sur le lien entre les inversions nominales et la théorie de l'information; Abeillé \& Godard 2004, sur le rôle du "poids" des constituants dans l'ordre; dans une perspective fonctionnaliste, Cornish $(2001,2005)$ à propos des inversions "locatives"; enfin, tout un pan de l'organisation de l'énoncé surtout repérable en français parlé a été étudié par l'école de BlancheBenveniste: voir C. Blanche-Benveniste (1996).

Dans le présent congrès, trois des communications en syntaxe touchent à ces questions (Tseng; Bonami \& Godard; Abeillé, Godard \& Sabio).

Dans de nombreuses théories actuelles, l'ordre des mots est distingué de la constituance. Ce n'était pas le cas autrefois, notamment dans les théories génératives, pour lesquelles l'ordre des mots découlait de l'ensemble des règles de formation de l'énoncé. Les modifications par rapport aux ordres conformes à la constituance étaient décrites par des modifications post-transformationnelles telles que Scrambling (Ross, 1986: 51).

La prise en compte de langues "non configurationnelles" comme le warlpiri a conduit à distinguer un ordre variable, soumis de fait à des facteurs pragmatiques, à la constituance ordonnée des langues configurationnelles (par exemple pour LFG: Bresnan, 2001: 9). Il en est résulté la prise en compte en syntaxe d'ordres régis par des facteurs communicatifs (E. Kiss, 1994).

Dans les théories génératives, l'ordre configurationnel est vu généralement comme le produit d'un paramètre sur la position des têtes, initiale (anglais) ou finale (japonais). A cela s'ajoutent des modifications qui doivent être motivées (scrambling, passif, inversion "stylistique"). L'analyse de Kayne (1994) basée sur une relation c-commande asymétrique suppose un ordre universel de type spécifieur-tête qui conduit à poser l'ordre SVO comme basique (Kayne, 1994: 35).

Le français moderne est typiquement une langue configurationnelle de type SVO, avec plusieurs fonctions affectées à l'ordre:

-fonctions grammaticales (distinction sujet /objet direct). 
- fonctions énonciatives: assez marginales en français moderne: l'interrogation totale de type VS mais avec sujet clitique; les incises; des résidus de type également VS (exclamatives, expressions figées). Le statut des sujets clitiques inversés est problématique en syntaxe (affixes ou éléments libres dans la dérivation?).

- fonctions informatives communicatives: l'ordre des compléments entre eux, qu'il y ait ou non une structure non marquée basique, est lié à des facteurs communicatifs: "poids" des constituants, focalisation, sachant que la dernière position syntagmatique liée est focus par défaut en français. L' inversion du sujet nominal "stylistique" (Kayne 1973) pourrait obéir à des facteurs pragmatiques particuliers. Ici aussi se pose le problème du statut morphologique ou syntaxique des inversions clitiques motivées par la présence d'un adverbe (type: Peut-être Jean viendra-t-il). Le statut communicatif des termes initiaux, soit dans le cas des inversions, les déclencheurs d'inversion clitique ou d'inversion du sujet nominal, est discuté: thème, focus, cadratif? De même, dans l'antéposition de l'objet devant un sujet (communication Abeillé, Godard, Sabio), l'une des constructions étudiées, le type à extraction, semble lié à la focalisation accompagnée d'une fonction de reprise discursive (voir plus loin). La dislocation, gauche ou droite, avec des fonctions discursives différentes, est également liée à des fonctions telles que la focalisation ou la thématisation.

Un autre angle d'étude est offert par la diachronie: selon les analyses admises, l'ancien français était une langue de type V2 dans les indépendantes avec probablement deux stades dans l'évolution, si on suit Rouveret 2004. Le statut du terme initial semble avoir été assez proche de celui des langues germaniques actuelles, soit topique soit focus, souvent aussi un adverbial "cadratif". Comment est-on passé au type SVO qui se manifeste nettement entre le 16e et le 17e siècle? Peut-on voir dans les "inversions" actuelles la survivance de structures de type TVS?

Avec les mêmes réserves que ci-dessus sur l'appartenance ou non à la syntaxe, le statut des clitiques a évolué (vers plus de cliticisation et une rigidification qui contraste plus ou moins avec le statut des autres clitiques romans). Comment le français s'est-il distingué sur ce plan des autres langues romanes? Faut-il analyser tous les clitiques comme relevant de la morphologie (Miller 1992)?

On se limitera ici aux questions relatives au français actuel. Après une brève présentation de la structure canonique des phrases, le type SVO, on examinera les constructions à inversion (VS, le plus souvent de type VOS avec sujet nominal), puis les constructions à objet antéposé (OSV). Faute de place, on ne parlera pas des disloquées.

\section{Ordre de base des constituants}

Le type SVO du français moderne standard écrit offre les propriétés suivantes:

Tout SN (non adverbial) directement placé devant le verbe fini est son sujet. Si on a la suite SV(initial)$\mathrm{SN}$, tout SN (non adverbial) directement à droite de $\mathrm{V}$ ou de la suite Aux-Vpp est objet direct sauf cas de construction impersonnelle avec un verbe non inaccusatif. Les constructions à $\mathrm{V}$ inaccusatif ont ici un sujet qui partage certaines propriétés de l'objet direct mais qui accorde à lui le verbe.

Les constructions orales offrent quelques possibilités supplémentaires sans qu'on y rencontre des ordres absolument absents de l'écrit (Blanche-Benveniste 1996: 109).

Les possibilités d'éléments $\mathrm{X}$ intercalés entre une structure de type SVO sont différentes selon les cas:

-entre $\mathrm{S}$ et $\mathrm{V}$, il est assez difficile sauf dislocation d'insérer un élément non clitique:

?Paul tous les soirs promène son chien

En particulier, l'insertion d'adverbes liés entre $\mathrm{S}$ et $\mathrm{V}$ est exclue, contrairement à ce qui est observé dans une autre langue SVO, l'anglais (Pollock 1989).

-si $V$ est auxiliaire: entre $V$ et Vpp, on peut insérer des adverbiaux: 
(4) Paul a pendant des heures joué avec son chien

L'insertion de groupes prépositionnels non adverbiaux semble difficile:

(5) ??Paul a de son patron obtenu une réduction

(6) *Paul avait à Marie donné ce livre

-entre $\mathrm{V}$ et $\mathrm{O}$, il n'y a pas de contrainte d'ordre indépendamment des choix pragmatiques et communicatifs:

(7) Paul donne à Marie un livre / Paul donne un livre à Marie

Sur l'ordre des compléments dans une langue SVO comme le français, il y a plusieurs explications possibles. Pour Hawkins $(1994 ; 1998)$, l'explication des ordres observés en performance est à rattacher à des choix liés à la complexité plus ou moins grande des compléments, les plus simples précédant les complexes. Selon lui, les explications par des différences de type communicatif dérivent de la complexité structurale.

Sans que cela soit une objection aux explications par des choix de performance liés à la complexité ou à des choix communicatifs, il semble qu'il y ait en français un ordre neutre de la séquence des compléments dans le SV, si on suit les propositions de Korzen 1996:

\section{SN (objet direct) SPrép (objet indirect) SAdv scéniques (temps /lieu) Autres SAdv}

La séquence linéarisée peut correspondre à deux types totalement différents de structuration. Dans les analyses génératives classiques à branchement binaire, il faut supposer une expansion à droite alliée à une dépendance structurale. L'hypothèse avancée par $\mathrm{C}$. Philips 2003 supposerait une expansion par accrétion sur des constituants syntagmatiques formés de façon successive de gauche à droite: une première association formerait ainsi un groupe V SN, ce groupe étant ensuite associé à un SPrép, et ainsi de suite.

L'argumentation détaillée de Korzen sur l'ordre neutre est basée sur les propriétés d'extraction des mots Qu- avec placement non final du sujet, on y reviendra plus loin.

Dans cet ordre neutre, les adverbiaux peuvent se placer selon les facteurs de poids, de complexité ou de focalisation, à des places diverses différentes de l'ordre neutre. Leur ordre relatif peut évidemment être significatif s'ils ont des relations prédicatives de portée l'un sur l'autre: c'est alors la précédence qui correspond à la position prédicative dominante:

(8) Luc n'a toujours pas répondu / Luc n'a pas toujours répondu

L'insertion d'un élément entre auxiliaire et participe passé n'est probablement pas neutre. Il faut distinguer certains éléments "légers" comme l'objet pronominal tout :

Il a tout cassé vs. ?Il a cassé tout

Les propriétés morphophonologiques ne suffisent pas pour expliquer cette position, qui semble rendue possible par un statut catégoriel particulier, peut-être semi-adverbial: on ne peut pas avoir:

$$
\text { *Il a ça cassé vs. Il a cassé ça. }
$$

Autre cas particulier: celui d'adverbes à statut de déterminant détaché, étudié par Obenauer 1983, avec une possible différence d'interprétation aspectuelle entre les deux constructions:

(11) Dans cette caverne, il a beaucoup trouvé de pièces d'or

(11') Dans cette caverne, il a trouvé beaucoup de pièces d'or (Obenauer 1983: 78) 
Il faut aussi distinguer l'auxiliaire à valeur de temps du passé de l'accompli utilisable avec d'autres prédicats que les participes passés: dans cette dernière interprétation, un SN sujet sémantique du prédicat peut précéder:

(12) J'ai cassé mon moteur

(12') J'ai mon moteur (de) cassé /...qui est cassé

Dans ce dernier cas, l'accompli n'a pas de valeur passée: par exemple on peut opposer:

(13) J'ai cassé mon moteur mais il est réparé

(13') *J'ai mon moteur cassé mais il est réparé

La différence tient sans doute au statut assez différent de avoir dans les deux constructions.

\section{Les constructions à inversion du sujet}

\subsection{Les inversions clitiques}

Il est souvent admis que les clitiques objets des langues romanes sont attachés morphologiquement au support verbal (dans la plupart des langues modernes actuelles, malgré certains cas d'attachements à d'autres supports: portugais par exemple, ou encore l'attachement secondaire en gascon aux particules initiales). L'attachement préverbal ou postverbal des clitiques objets encore observable dans les langues romanes du sud pour les formes non finies du verbe a disparu depuis longtemps du français, et la possibilité de placer les clitiques objets sur un auxiliaire distinct des auxiliaires de l'accompli et du causatif en faire est totalement archaïque en français:

(14) *Il le leur veut donner

La principale question relative à l'ordre syntaxique des constituants est celle que pose le pronom faible sujet, cliticisé en français, d'abord en position postverbale (c'est déjà fait en français médiéval selon Skårup), puis en position préverbale à la fin de la période de l'ancien français. Les analyses de ce phénomène varient: soit le clitique est décrit comme un élément morphologique sans incidence sur la syntaxe de la phrase en français moderne: sa postposition serait morphologique (Morin 1979), soit il est décrit comme un terme occupant une position syntaxique distincte, analogue à celle du sujet nominal avec une cliticisation postsyntaxique, au niveau phonologique (Kayne 1983, Rizzi \& Roberts 1989, position identique dans l'analyse diachronique de Dufresne \& Dupuis 1996). L'analyse proposée par Rizzi \& Roberts alignait la syntaxe du français sur celle des langues germaniques avec une montée du verbe audelà de la position du sujet occupée par le clitique, donc en C. Cette analyse est insatisfaisante pour plusieurs raisons, notamment la difficulté de décrire de façon non ad hoc les constructions à sujet nominal coexistant avec le sujet clitique (on parle dans ce cas d'inversion complexe): le sujet nominal devrait occuper la position de spécifieur de $\mathrm{C}$, mais se trouve alors en concurrence avec la possibilité de trouver aussi un mot Qu-:

Depuis quand Jean la connaît-il? (Kayne 1994: 44)

Enfin, on n'a pas en français (ou plus exactement, on ne trouve plus depuis le 13e siècle) des constructions interrogatives de type:

(16) *Est Jean venu?

Ces deux raisons ont conduit Kayne à abandonner l'hypothèse d'un mouvement vers C (Kayne 1994: 44). Depuis les propositions de Rizzi, 1997, l'hypothèse posant la présence de plusieurs têtes fonctionnelles à la périphérie gauche de la proposition permet d'expliquer les phénomènes d'inversion en subordonnée ou comme en ancien français l'existence de constructions de type V2 après une conjonction (Rouveret 2004: 227). 
La dérivation des inversions clitiques dans une théories HPSG est esquissée dans la communication de J. Tseng. Cette communication se limite cependant au problème du sujet, alors que l'inversion clitique implique la syntaxe d'un élément déclencheur en position précédant le sujet, soit adverbe, soit pronom Qu-, soit segment en discours direct dans les incises:

(17) Peut-être Jean est-il arrivé vs. *Jean est-il peut-être arrivé.

(18) Où Jean est-il allé? vs. *Jean est-il allé où?

(19) "Viens ici", me dit-il. vs. *Me dit-il: "Viens ici".

L'absence de ce déclencheur en contextes antérieur et postérieur vides ${ }^{1}$ impose une interprétation interrogative, il faut donc poser impérativement un élément vide dans ce cas, dans le paradigme des autres déclencheurs:

(20) Jean est-il parti *(?)

On peut donc analyser l'inversion clitique comme un phénomène de rection un peu particulier, distinct de la subordination: l'interprétation énonciative de la séquence ( $S N)$-il dépend du déclencheur, un déclencheur vide aboutissant à une interprétation interrogative (Muller 1996: 76). La présence ou absence d'un SN intercalé (dans les contextes à inversion complexe) est transparente:

$$
\text { Peut-être (Jean ) est-il arrivé }
$$

Les déclencheurs sont spécifiques à ce type d'inversion, même s'il y a une interférence avec ceux de l'inversion du sujet nominal (voir plus loin). Pour l'inversion complexe, il s'agit: des mots Quinterrogatifs, d'adverbes, soit de modalisation énonciative ( peut-être, sans doute, assurément...) soit à fonction de connecteurs (aussi, ainsi,...) avec un énoncé antérieur, quelques adverbes à orientation négative (rarement, difficilement), et des constructions à corrélation, à interprétation hypothétique:

(22) Quand Jean est-il venu?

(23) Assurément Jean est-il arrivé

(24) ...Aussi Pierre est-il intervenu

(25) Rarement trouvait-on le curé à son logis (M. Aymé, La Table-aux-Crevés, Pléiade, 1, 253)

(26) A peine Marie était-elle sortie que Jean est entré

(27) Jean sort-il que Marie entre aussitôt

(28) Une discussion venait-elle à s'élever au bureau, il ne s'en mêlait point que son avis ne fût sollicité...(M. Aymé, Aller-retour, Pléiade, 1, 113).

L'inversion clitique se confond avec l'inversion du sujet nominal dans les contextes suivants:

-Des emplois au subjonctif avec auxiliaire modal:

$$
\text { Puisse-il (Puisse Jean) réussir! }
$$

-Les incises après discours direct:

(30) Salut! dit-il (...dit Jean)

-Après que pronom interrogatif:

Que fait-il? (Que fait Jean?)

\subsubsection{L'inversion du sujet clitique en subordonnée}

La plupart du temps, on trouve l'inversion clitique dans les indépendantes et les principales, ce qui a été interprété comme l'obligation que le constituant $\mathrm{C}_{0}$ soit vide et non régi par un terme recteur (pronom 
relatif ou interrogatif par exemple). Il est cependant possible de trouver cette inversion clitique en subordonnée:

(32) Il a tellement envie de venir que peut-être viendra-t-il quand même nous voir.

(33) C'est que peut-être ne s'agissait-il pas d'une nouvelle manière de peindre, mais, plus fondamentalement, d'une nouvelle manière de faire...(J. Cassou, Panorama des arts plastiques contemporains, 1960, 171, Frantext). ${ }^{2}$

(34) Tant de filles honnêtes sont devenues de malhonnêtes femmes, que peut-être serai-je un exemple contraire (Diderot, Jacques le Fataliste, 1784, Frantext, 647).

(35) Ane et cheval, ils travaillaient chacun de leur côté, et le soir, à l'écurie, se retrouvaient si harassés qu'à peine, avant de s'endormir, avaient-ils le temps d'échanger quelques plaintes sur la dureté de leurs maîtres. (M. Aymé, Contes du chat perché, Pléiade, 2, 1013).

Cette situation n'est que le prolongement en français moderne d'une particularité du français: contrairement à d'autres langues V2, qui ont une structure différente en principale et en subordonnée, le français a toujours eu la possibilité de construire ses subordonnées sur les types syntaxiques des indépendantes: dès l'ancien français (seconde moitié du XIIe siècle), la subordonnée adopte le modèle des indépendantes avec possibilité (très minoritaire) d'inverser le sujet (Skårup 1975: 515).

L'inversion du clitique sujet pose la question de la nature du sujet (Tseng, dans sa communication). Il ne semble pas, malgré quelques vues discordantes qu'il y ait à discuter de la fonction du $\mathrm{SN}$ accordé au clitique: même si à l'origine c'était indiscutablement un nom en extraposition ${ }^{3}$, séparé par une pause du sujet, ou même antéposé parfois au déclencheur, son intégration comme sujet découle de la possibilité de voir les indéfinis dans cette position avec accord au clitique:

Peut-être quelqu'un est-il arrivé

alors que les indéfinis de ce type ne sont pas thématisables librement:

(37) *Quelqu'un, est-il arrivé?

Autre argument: l'accord avec un pronom neutre non humain, exclu en français moderne en cas de thématisation:

(38) Cela est-il vrai?

(39) *cela, est-il vrai? vs. Cela, est-ce vrai?

Il faut donc voir dans ce SN un sujet, et d'autre part aucun argument ne permet de lui fixer une position syntaxique distincte de celle des sujets nominaux des énoncés sans clitique.

La nature du sujet tient à l'accord. Il semble qu'on puisse admettre les possibilités suivantes:

-il et son paradigme sont imposés par l'utilisation d'un temps fini. cette particule est donc à interpréter comme une marque de temps. Sans argument à construire (verbes impersonnels), on a l'interprétation impersonnelle, avec antéposition ou postposition selon les mêmes critères que dans les constructions avec accord.

-L'accord est indépendant de toute considération sémantique: il s'adapte évidemment à la pluralité obtenue par coordination:

(40) Pierre et Marie sont-ils venus?

-Les emplois en pronom personnel sont dus à l'absence de réalisation du pronom fort après accord: il ne reste que le clitique avec la sémantique des pronoms personnels cette fois. Ce phénomène doit être étendu à $c e$ qui rend inutile la réalisation phonologique d'un cela, ça, ou a on qui correspond à un SN humain indéterminé. 
La structure des constructions à inversion clitique pourrait suivre le modèle suivant, basé sur l'hypothèse d'une position distincte du sujet clitique basique (il impersonnel):

$$
\text { Déclencheur } \quad \text { (Sujet nominal) } \quad V \text {-il } \quad[(e)]_{V} \ldots
$$

Cela suppose, pour les phrases sans clitique, un mécanisme d'élagage du clitique lorsque le sujet nominal est réalisé sans inversion clitique, et donc la possible réalisation non standard de phrases à double sujet à gauche, actuellement bien attestée en français familier (Zribi-Hertz 1994) et dans les corpus de français parlé. Par exemple, Auger 1996:

(41) mon frère le plus vieux il jouait du violon

(42) en campagne, quand quelqu'un il dansait...(Auger 1996: 25)

Il n'en découle pas nécessairement que le français standard soit analysable de même (Auger 1996: 39). Position identique dans Zribi-Hertz 1994.

\subsubsection{L'inversion du sujet nominal}

Il s'agit de diverses constructions à sujet nominal (non clitique) accordé au verbe qui le précède. Cette inversion a été nommée "inversion stylistique" (Kayne 1973). Il existe de nombreuses études depuis une trentaine d'années sur ce sujet. L'article de Kampers-Mahne, Marandin, Drijkoningen, Doetjes \& Hulk (2004) distingue plusieurs types:

1. L'inversion dans les contextes d'extraction (questions partielles, relatives, clivées):

(43) Où est allée Marie? Je me demande où est allée Marie.

(44) La personne qu'a rencontrée Pierre est ma cousine.

(45) C'est dans cette maison qu'est né Victor Hugo.

2. L'inversion inaccusative (Marandin 2003), liée à des verbes spécifiques (verbes de mouvement, verbes avec auxiliaire être, passifs); elle est observable dans deux classes distinctes de contextes:

-complétives:

(46) Je voudrais que soient distribués ces prospectus (Kampers-Mahne et al., 2004: 557).

(47) On eût dit que traînait dans la pièce quelque chose de cette atmosphère lourde...(Gracq, Le rivage des syrtes, 32, Frantext).

-indépendantes avec ou sans adverbe introducteur:

(48) A ce moment-là se fit entendre un bruit strident.

(49) Entre alors notre gardien avec de la nourriture.

Elle pourrait être liée à un statut particulier du sujet inversé, attaché à une position "profonde" d'objet direct distincte de la position standard du sujet dans les analyses génératives, comme spécifieur et non comme complément du verbe. En témoigne la propriété de ce type de sujet inversé de pouvoir lier un en au sujet postposé quantifieur:

(50) (des examens) Il faudrait qu'en soient reportés plusieurs.

(51) (des hommes) Alors en entrèrent trois / En entrèrent trois.

(exemples (17) de Bonami-Godard (2001: 123)). 
3. L'inversion "elaborative" (Kampers-Mahne et al.) ou "à focalisation forte" (Kayne \& Pollock 2001): il s'agit d'inversions à sujet focalisé, souvent lourd, incluant les listes, et sans contrainte d'utilisation de la première place:

$\left(52^{\circ} \quad\right.$ Ont obtenu leur licence les étudiants suivants: Pierre Dupont, Marie Dubois...

Le sujet pluriel cataphorique n'est pas obligatoire: la liste peut suivre immédiatement le verbe, constituant ainsi le seul cas connu de sujet syntaxique disloqué:

(53) Ont obtenu leur licence: Pierre Dupont, Marie Dubois...

Un objet est possible comme on le voit ci-dessus, il est alors toujours placé avant le sujet (plus généralement, le sujet est final dans ce type):

(54) Rendront un devoir supplémentaire tous les élèves qui ont échoué (Kampers-Mahne et al. 2004: $559)$.

4. Il faut ajouter à ces inversions étudiées par Kampers-Mahne et al. les inversions à topique adverbial ou adjectival initial parmi lesquelles les inversions locatives étudiées par F. Cornish (2001), A. Borillo (2006), C. Fuchs (2006): un adverbial de lieu, parfois de temps, introduit une construction à sujet le plus souvent, mais pas obligatoirement, final:

(55) Sur la place se dresse la cathédrale.

(56) A midi sera organisé un apéritif dans la salle des fêtes.

(57) Il y a trois siècles, ici naissait Blaise Pascal (Borillo 2006: 25)

Sans doute n'est-ce qu'un sous-type fréquent d'inversions à adverbial initial focalisé:

(58) Ainsi se termina le spectacle.

(59) Avec Autun se distingue Alésia par le nombre d'ateliers découverts. (Dossiers d'archéologie, n³16, sept. 2006, 52).

ou de constructions à adjectif initial:

(60) Nombreux sont les gens qui se plaignent.

(61) Frugal est leur quotidien: du thé au beurre, une poignée de farine d'orge grillée...(Tibet, dans le Monde Voyages, Mars 1992, 16).

Cet ensemble de constructions ressemble par le type de verbe aux constructions inaccusatives mais a une spécificité dans l'analyse informationnelle du verbe: celui-ci est soit faiblement informatif, soit de signification réduite dans la construction à inversion locative (cf. plus loin).

5. Il faut ajouter aussi les constructions à incise. Bonami \& Godard (communication à cmlf) distingue deux types, dont l'un avec inversion est obligatoire, qu'elle soit nominale ou clitique, lorsque l'incise est un "ajout" à une citation considérée comme une "tête":

(62) Je ne vous crois pas, s'emporta enfin la femme. (Dorgelès, dans Grevisse, §1352).

Selon ces auteurs, ce type d'incise obéit aux critères des structures à extraction, le critère essentiel étant la possibilité de régir la citation par un verbe enchâssé:

(63) "Je n'en peux plus" semblait croire pouvoir dire Paul. (op. cit. ex. 33d).

Enfin, contrairement à d'autres langues romanes comme l'italien, le français ne connaît pas d'inversion du sujet nominal en réponse, avec focalisation (Marandin 2003) ou sans (Lambrecht, 1994):

(64) Ha telefonato Gianni (Lambrecht 1994)

(65) *A téléphoné Jean. 
Le statut du sujet inversé est discuté par divers auteurs. Selon Bonami-Godard 2001, sept propriétés caractérisent le sujet inversé (en laissant de côté les sujets des inversions inaccusatives, plus proche de l'objet direct)

a. Le lien cataphorique réfléchi - sujet: A qui s'est présenté Paul?

b. Pas de lien entre en et un quantifieur sujet: *Celui qu'en ont déjà lu plusieurs.

c. Pas de construction à distance $\mathrm{Q}$ de $\mathrm{N}$ : *C'est un livre qu'ont beaucoup lu de gens.

d. L'extraction de combien est possible: Combien pensez-vous que viendront de ministres? vs. * Combien pensez-vous que de ministres viendront?

e. Le sujet peut être construit avec de négatif: Une maison où ne viennent plus jamais d'enfants.

f. Les quantifieurs nus sont exclus, sauf s'ils sont possibles comme objets directs: *Une hypothèse à laquelle s'intéressent tous / plusieurs/ beaucoup vs. Une hypothèse à laquelle tous/ plusieurs/ beaucoup s'intéressent.

g. L'accord ne se fait qu'en nombre: *L'appartement qu'habitiez Claire et toi à l'époque vs. L'appartement que Claire et toi habitiez à l'époque. (Exemples de Bonami \& Godard 2001).

Dans le détail, les inversions ont un fonctionnement assez différent selon les types. Sur l'inversion locative, A. Borillo 2006: 33, note que le prédicat est "léger" d'un point de vue informationnel, au point de perdre du sens, comme le montrent les exemples suivants:

(66) Dans l'armoire étaient rangées les chaussures.

(66') Dans l'armoire, les chaussures étaient rangées. (Cité par Nølke 1995, ex; (33), l'exemple originel étant d'A. Borillo; repris dans Cornish 2001: 110)

Le sens est différent: dans la structure à sujet final, le verbe localise simplement les chaussures; dans la phrase à sujet préverbal, le verbe focalisé prend son sens plein et "rangé" a un sens qualitatif. Cela peut conduire à un inacceptabilité si le verbe final n'est pas susceptible de focalisation, comme dans:

(67) *Dans l'armoire, les chaussures se trouvaient (Nølke 1995, ex. (36))

D'autres effets peuvent apparaître, par exemple (dans Cornish 2001: 106):

Dans ce bureau travaillent quatre personnes.

(68') Dans ce bureau quatre personnes travaillent.

La phrase à inversion prend un sens habituel, alors que la phrase à sujet préverbal a un sens actualisé.

On peut ajouter à ces exemples la paire suivante, avec un verbe d'action:

(69) Dans l'armoire, les chaussures sont cirées.

(69') Dans l'armoire sont cirées les chaussures.

La seconde montre comment l'inversion s'associe au sens habituel pour localiser l'action, donnant à la phrase le sens bizarre du lieu où se fait l'action. La phrase à verbe final est interprétée comme un accompli descriptif avec le sens qualitatif déjà vu plus haut.

L'inversion à extraction pose d'autres problèmes. Le sujet peut plus facilement n'être pas final, et sa position semble être préférentiellement à l'endroit où le terme extrait avait sa position in situ (Korzen, 1992; 1996). L'analyse de Korzen propose une explication à la position du sujet: celui-ci doit se placer, sauf facteurs supplémentaires comme la lourdeur particulière de certains syntagmes, juste après la position d'un terme Qu- in situ, et l'ensemble du verbe et des compléments allant jusque là constitue une unité pragmatique distincte de la constituance syntagmatique, qu'elle nomme unité prédicative minimale. Ainsi, sachant que les adverbiaux de temps précèdent par défaut les adverbiaux de lieu, on expliquera le contraste suivant: 
(70) A quelle heure ferment les magasins en France?

unité prédicative: ferment à quelle heure.

(71) *Dans quel pays ferment les magasins à huit heures? (Korzen 1996: 61)

unité prédicative: ferment à huit heures dans quel pays.

On dira, conformément à cette analyse:

(71') Dans quel pays ferment à huit heures les magasins?

La valeur empirique de l'analyse de Korzen est admise par Kayne \& Pollock 2001 et incorporée aux transformations multiples produisant dans leur cadre la structure à inversion par une série de mouvements à gauche.

La concurrence, à droite du verbe, de l'objet direct et du sujet pose des problèmes spécifiques. L'objet direct précède en principe le sujet:

(72) Le dieu (Hugo) est entouré d'êtres féminins. Il y en a tout un canapé, parmi lesquels fait les honneurs du salon une vieille femme aux cheveux d'argent... (Goncourt, Journal, 10-12_1870).

Certains cas où le sujet précède l'objet s'expliquent bien par les propositions de Korzen:

(73) (il les disperse) ainsi que fait un vent d'orage les épis. (Genevoix, Ceux de 14, Frantext, 42)

L'extraction implique un adverbial de manière qui précède l'objet: un vent d'orage disperse ainsi les épis. L'ordre inverse serait impossible ici:

(74) * ainsi que fait les épis un vent d'orage

Autre cas, l'extraction d'un locatif circonstanciel qui peut être naturellement interprété comme précédant l'objet:

(75) C'est chez eux que trouvent leurs invalides la fameuse "loi de l'offre et de la demande" (L. Febvre, Combats pour l'histoire, Frantext, 173)

$=$ leurs invalides trouvent [là] la fameuse loi de l'offre et de la demande.

Il existe pourtant de nombreux cas d'inacceptabilité qui ne s'expliquent pas par la théorie de Korzen. Par exemple:

(76) *A qui a donné ce livre Jean? (Kayne et Pollock 2001, ex. (167))

comparé à l'exemple suivant, plus acceptable:

(77) ?Qu'a donné à Marie Jean? (ex. (166))

Sur un exemple presque identique:

(78) *L'enfant auquel donnera ce livre Paul (Bonami \& Godard, ex (1b) et (71b))

Il n'y a pas d'exclusion de la fonction objet direct, puisque les objets clitiques sont toujours possibles:

(79) J'éprouve un peu du tressaillement qui saisit M. de Portebize quand les lui décrit M. d'Oriocourt (M. Proust, dans Damourette \& Pichon, §1590).

Bonami \& Godard posent une contrainte de domaine excluant la succession de deux GN non prédicatifs ${ }^{4}$ non locatifs non lourds. De fait, les GN lourds sont admis dans ce cas en position finale après un objet direct:

(80) C'est finalement à Marie qu'a présenté son projet l'étudiant que Paul avait refusé de recevoir. (B \& G, op. cit.)

Exemple réel: 
(81) Où puisait donc sa sagesse improvisée cette catalane mercenaire, qui n'était jamais qu'un mercanti de la littérature? (J. Donoso, Le jardin d'à-côté, Frantext).

Cette analyse ne me semble pas entièrement satisfaisante, puisqu'il me semble qu'on peut dire facilement:

Où donc cueillent des fleurs les parisiens?

A qui donc offrent des fleurs les maris, à la Saint-Valentin?

\subsubsection{Les inversions à longue distance}

Il s'agit de constructions dans lesquelles le sujet suit des termes régis par le verbe principal dans une autre proposition, sans être nécessairement final. On trouve en effet des sujets inversés entre compléments régis, sans limite, que ce soit des compléments infinitifs ou des verbes finis:

(84) Quel genre de cadeau veut offrir Marie à Jean-Jacques? (Kayne \& Pollock 2001 ex. (159)).

(85) Quelle maison veut que j'achète Jean-Jacques? (idem ex. (157)).

(86) Une chance que trouvent toujours plus ou moins qu'on a les femmes dont on devient l'amant... (P. Léautaud, Le petit ouvrage inachevé, 30, Frantext; dans MUller 2002).

Avec un verbe fini régi, la possibilité de trouver un sujet non final semble plus réduite:

*Quelle maison veut que j'achète Jean-Jacques à Marie?

Ces constructions, comme on le voit, ne respectent pas la constituance en syntagmes. Pour Bonami \& Godard (2001: 133) "toutes les structures proposées pour l'inversion simple sont inadéquates pour l'inversion longue". Leur démonstration se base sur une analyse par extraposition droite du sujet, elle même suivie d'une extraposition droite plus haut placée du terme final. Cette extraposition doit être non bornée, ce qui contrevient à la règle établie par Ross (1967/ 1986) selon laquelle les extrapositions droites sont des dépendances bornées. Par conséquent, ces auteurs proposent de distinguer l'ordre et la constituance.

Pour Kayne \& Pollock, les déplacements multiples à gauche supposent un déplacement particulier du terme qui apparaîtra en position finale; deux solutions alternatives sont présentées: soit ce terme est déplacé par pied-piping avec le mot Qu-, soit il est déplacé ultérieurement et séparé de son recteur par 'topicalisation' (section 16). Il faut en outre une transformation particulière, IP movement, pour déplacer le verbe et les verbes dépendants, tensés ou non.

Les constructions avec régis à l'infinitif présentent des possibilités contrastées. Les cas les plus simples sont ceux d'infinitifs contrôlés par le même sujet, avec une série non limitée de verbes régis:

Qu'a oublié de raconter Luc à Marie?

Du point de vue de l'ordre, et probablement aussi pour les propriétés phonologiques et prosodiques, "a oublié de raconter" est traité comme une unité, qui peut ici correspondre à une extension de la notion pragmatique d'unité prédicative minimale de Korzen 1983. Lorsque le contrôle de l'infinitif est exercé par un autre actant, des contraintes supplémentaires apparaissent qui peuvent contraindre le sujet inversé à être final. Bonami \& Godard 2001 opposent:

*La personne que m'a convaincu de présenter le patron du labo à Marie.

La personne que m'a convaincu de présenter à Marie le patron du labo.

Il fait sans doute poser que l'unité prédicative minimale incluant un sujet sémantique de verbe à l'infinitif (son contrôleur) doit aussi incorporer tous les compléments rattachés à ce verbe (Muller 2002).

D'autres constructions de ce type sont à prendre en compte, en particulier:

-les coordonnées à sujet à droite commun: 
(90) Peu importe dès lors ce que valent et ce que deviennent les fruits de la terre. (Teilhard de Chardin, Le milieu divin 38, Frantext)

-les dépendantes à sujet commun, qui semblent acceptables dans certains cas:

(91) C'est ce que dit que fait Mimi quand elle a des visites. (Damourette \& Pichon, §1589)

(92) Ce qu'a dit que ferait Luc à Marie est inquiétant

\section{Les compléments antéposés}

Le français, surtout oral, connaît une structure marquée de type OSV. Elle a été analysée à partir des corpus de français parlé (cf. par exemple Blanche-Benveniste 1996: 112), mais elle avait déjà été signalée par Blinkenberg (1928: 164). Elle fait l'objet d'un traitement macro-syntaxique, avec la distinction de deux constructions, dans Sabio 2006. C'est aussi l'objet de la communication d'Abeillé, Godard \& Sabio au congrès, avec une argumentation qui conduit ces auteurs à distinguer deux constructions, l'une d'un type disloqué à complément nul anaphorique, dans lesquelles le "complément" initial est un thème:

(93) Les conjugaisons j'aimais bien (auteurs cités, ex. (1))

l'autre à extraction, avec un complément non thème:

(94) et là, tu sais ce qui lui est arrivé -une antenne ils lui ont jeté à la tête...(ex. (15a))

(95) Trois heures il avait de retard, le train. (ex. (7b))

La propriété principale permettant dans ce dernier cas de parler d'extraction est l'impossibilité de supprimer le complément antéposé, à la différence de la première construction.

La seconde construction obéit à des contraintes particulières contextuelles, qui conduit les auteurs à parler d'extraction dialogique. Même avec un indéfini, une reprise est possible:

(96) Mon père il va m'acheter un petit mouton + un petit mouton il va m'acheter. (Ex. (15c)).

\section{Conclusion}

Très sommairement, faute de place, je me contenterai de souligner quelques points saillants parmi les principales évolutions de ces recherches: la distinction faite de plus en plus souvent entre ordre et constituance; la prise en compte croissante de la structure de l'information dans les structures non canoniques; le développement des investigations syntaxiques sur le français parlé.

\section{Références bibliographiques}

Abeillé A., Godard (2004). De la légèreté en syntaxe. Bulletetin de la Société de Linguistique de Paris, 69-106.

Auger J. (1996). Subject-Clitic Inversion in Romance: A Morphological Analysis. In: C. Parodi, C. Quicoli, M. Saltarelli \& M.L. Zubizarreta (Eds): Aspects of Romance Linguistics. Georgetown U.P. , 23-40.

Blanche-Benveniste C. (1996). Trois Remarques sur l'ordre des mots dans la langue parlée. Langue française 111,109-119.

Blinkenberg A. (1928). L'ordre des mots en français moderne. Première partie. Copenhague.

Bonami O., Godard D. (2001). Inversion du sujet, constituance et ordre des mots. In: Marandin J.M. (éd): Cahier Jean-Claude Milner. Paris: Verdier, 117-174.

Bresnan J. (2001). lexical-Functional Syntax. Oxford: Blackwell. 
Cornish F. (2001). L'inversion 'locative' en français, italien et anglais: propriétés syntaxiques, sémantiques et discursives. Cahiers de grammaire, 26, 101-123.

Cornish F. (2005). A crosslinguistic study of 'locative inversion': Evidence for the Functional Discourse Grammar model. In: C. de Groot \& K. Hengeveld (Eds.): Morphosyntactic Expression in Functional Grammar. Berlin: Mouton-de Gruyter, 163-202.

Damourette J \& E. Pichon (1911-1940). Des mots à la pensée. Essai de grammaire de la langue française. Paris: D'Artrey.

Dufresne M. \& F. Dupuis (1996). Between Syntax and Phonology: Subjects Pronouns in French. In: C. Parodi, C. Quicoli, M. Saltarelli \& M.L. Zubizarreta (Eds): Aspects of Romance Linguistics. Georgetown U.P., 195-211.

Fuchs C. (2006). Locatif spatial initial et position du sujet nominal. Linguisticae Investigationes XXIX, 61-74.

Gerdes K. \& C. Muller (Eds) (2006). Ordre des mots et topologie e la phrase française. Linguisticae Investigationes XXIX-1.

Gerdes K. \& C. Muller (2006). Présentation. In: Gerdes K \& C. Muller 'Eds), 1-10.

Hawkins J.A. (1994). A Performance Theory of Order and Constituency. Cambridge: Cambrige U.P.

Hawkins J.A. (1998). Some issues in a performance theory of word order. In: A. Siewierska (ed.): Constituent Order in the Languages of Europe. Berlin: Mouton de Gruyter, 729-781.

Herslund, M. (2006). La topologie du français à la lumière de deux autres systèmes: le danois et l'ancien français. Linguisticae Investigationes, XXIX, 103-112.

Kayne R.S. (1973). L'inversion du sujet dans les propositions interrogatives. Le Français moderne, 41, 10-41.

Kayne R.S. (1983). Chaînes, catégories extérieures à S et inversion complexe en français. Langue française 58, 3665 .

Kayne R. S. (1994). The Antisymmetry of Syntax. Cambridge (Mass.): the MIT Press.

Kayne R.S. \& J.Y. Pollock (2001). New Thoughts on Stylistic Inversion. In: A. Hulk \& J.Y. Pollock (Eds): Stlistic Inversion in Romance and the Thory of Universal Grammar. Oxford: Oxford U.P., 107-162.

Kampers-Mahne B., Marandin J.M., Drijkongen F. Doetjes J. \& A. Hulk (2004). Subject NP Inversion. In: F. Corblin \& H. de SWart (Eds): Handbook of French Semantics. Stanford: CSLI Publications, 553-579.

É. Kiss, K. (ed) (1994). Discourse Configurational Languages. Oxford: Oxford U.P.

Korzen, H. (1992). The Predicative Unit and Subject-Verb Inversion in Modern French. In: M. Herslund (ed): Word Order, Copenhagen Studies in Language, 15, 65-123.

Korzen, H. (1996). La place du sujet non clitique dans la construction inversée. Langue française, 111, 59-82.

Lambrecht K. (1994). Information Structure and Sentence Form. Cambridge: Cambridge U.P.

Marandin J.M. (2003). Inversion du sujet et discours dans les langues romanes. IN: D. Godard (éd): Les langues romanes. Problèmes de la phrase simple. Paris: CNRS Editions, 345-392.

Miller P. (1992). Clitics and Constituents in Phrase Structure Grammar. new York: Garland.

Morin Y.C. (1979). There is no Inversion of Subject Clitics in Modern French. Ms., Université de Montréal.

Muller C. (1996). La subordination en français. Paris: Armand Colin.

Muller C. (2003). Inversion finale du sujet ou inversion postverbale? Cahiers de grammaire, 27, 121-145.

Nølke, H. (1995). Utterance focus: elements of a modular theory. Copenhagen Studies in Linguistics, 18, 74-108.

Obenauer H.G. (1983). Une quantification non canonique: la quantification à distance. Langue française, 58, 66-88.

Phillips C. (2003). Linear Order and Constituency. Linguistic Inquiry, 34, 37-90.

Pollock J.Y. (1989). Verb Movement, Universal Grammar and the Structure of IP. Linguistic Inquiry, 20, 365-424.

Rizzi L. (1997). The Fine Structure of Left Periphery. In: L. Haegeman (éd): Elements of Grammar. Handbook of Generative Syntax. Dordrecht: Kluwer, 281-337. 
Rizzi L. \& I. Roberts (1989). Complex Inversion in French. Probus, 1, 1-30.

Ross J.R. (1986). Infinite Syntax. (Edition de Constraints on Variables in Syntax, 1967). Norwood: Ablex.

Rouveret A. (2004). Les clitiques pronominaux et la périphérie gauche en ancien français. Bulletin de la Société de Linguistique de Paris, 99, 181-237.

Sabio F. (2006). L'antéposition des compléments dans le français contemporain. Linguisticae Investigationes, XXIX, $173-182$.

Skårup, P. (1975). Les premières zones de la proposition en ancien français. Copenhague: Akademisk Forlag.

Zribi-Hertz A. (1994). La syntaxe des clitiques nominatifs en français standard et en français avancé. Travaux de linguistique et de philologie, 32, 131-148.

1 Marginalement, on trouve parfois des interprétations exclamatives:

Est-elle jolie!

En contexte à subordination droite, on a une interprétation hypothétique:

Viendrait-il que ça ne m'étonnerait pas.

2 J'ai examiné 879 occurrences de la construction que peut-être dans Frantext: sur ce nombre, il y a 56 cas d'inversion du sujet clitique en subordonnée, ce qui est loin d'être négligeable.

3 Pour preuve la réflexion de Palsgrave, dans "L'éclaircissement de la langue française" (1530/2003: 492, édition H. Champion) cité dans la traduction moderne: "En français, ils mettent d'abord le substantif, et ils posent ensuite la question, ainsi: le roy ou s'en va il? Charles ou est il? ma robe est elle nette, mon cheval l'avez vous sellé? Pour lui, il s'agit encore d'une structure à thématisation. On le voit, le nom a pu précéder un terme Qu-. L'inversion complexe est attestée à la fin du XVe siècle dans les interrogatives, et à la fin du XVIe siècle dans les constructions à adverbe en tête.

4 Les GN prédicatifs sont attributs, ou encore compléments de verbes supports: par exemple:

Paul qu'avait nommé président du Club la commission unanime (Bonami \& Godard 2001: )

Plusieurs des exemples qu'on a trouvés en corpus correspondent de fait à des verbes supports: Frantext)

C'est ainsi que prit sa plein efficacité la théorie de l'Einführung... (R. Huygue, Dialogue avec le visible, 432,

Alors se précisa et prit tout son essor le rôle de la mer dans la vie nationale des Grecs. (R. Toutain, L'économie antique, Frantext) 